\title{
Through the looking-glass: Objects in the mirror are less real
}

\author{
Preeti Sareen • Krista A. Ehinger • Jeremy M. Wolfe
}

Published online: 19 November 2014

(C) Psychonomic Society, Inc. 2014

\begin{abstract}
Is an object reflected in a mirror perceived differently from an object that is seen directly? We asked observers to label "everything" in photographs of real-world scenes. Some scenes contained a mirror in which objects could be seen. Reflected objects received significantly fewer labels than did their nonreflected counterparts. If an object was visible only as a reflection, it was labeled more often than a reflected object that appeared both as a reflection and nonreflected in the room. These unique reflected objects were still not labeled more often than the unique nonreflected objects in the room. In a second experiment, we used a change blindness paradigm in which equivalent object changes occurred in the nonreflected and reflected parts of the scene. Reaction times were longer and accuracy was lower for finding the changes in reflections. These results suggest that reflected information is easily discounted when processing images of natural scenes.
\end{abstract}

Keywords Mirrors · Change blindness · Indoor scenes · Scene perception

We use visual information reflected from mirrors for various everyday purposes, such as grooming every morning or gauging traffic while driving. The image of an object (the reflection) formed by a plane mirror is optically identical to the actual object. However, of course, a reflection is not the same as a real object. The world through the looking glass is inaccessible (apologies to Lewis Carroll). Is the unreality of the looking-glass world reflected in the way that we interact with pictures that do and do not contain reflected objects?

P. Sareen $(\varangle) \cdot$ K. A. Ehinger $\cdot$ J. M. Wolfe

Visual Attention Lab, Harvard Medical School, Brigham \& Women's Hospital, 64 Sidney St. Suite. 170, Cambridge 02139, MA, USA

e-mail: psareen@partners.org
Most of the work on the perception of mirror images has focused on different problems, such as whether other species can recognize themselves in a mirror (Gallup, 1977; Prior, Schwarz, \& Güntürkün, 2008) or whether mirrors really reverse images left to right while not reversing them top to bottom (Corballis, 2001; Navon, 2001, 2002).

Mirrors produce some perceptual distortions. For instance, the impression of depth can be enhanced in mirrors (Higashiyama \& Shimono, 2012). A substantial body of recent work has been concerned with the difficulty that we have in the understanding of mirror reflections. Although we understand the basics of reflection, naïve beliefs about optics, like naïve physics (Proffitt, 1999), lead us to make errors. For instance, does an object appear smaller if it is farther from the mirror? People think that the answer is yes, even though the real determinant is the distance from the object to the observer. If that does not change, the distance to the mirror does not matter (Bertamini, Lawson, \& Liu, 2008). In the same vein, people are undisturbed by paintings with physically impossible depictions of people looking at themselves in mirrors (Bertamini, Latto, \& Spooner, 2003; Bertamini, Lawson, Jones, \& Winters, 2010).

The contribution of objects seen in mirrors to our understanding of a scene - the topic of the present study — does not appear to have been the subject of much prior research. It is unclear whether visual information from a mirror is treated differently when parsing a scene or when performing a visual task such as search. One might expect mirror information to be down-weighted in some visual-processing tasks: For example, when trying to interpret the shape of a room, people should treat mirrors as flat surfaces and ignore the 3-D depth cues from the reflection - though, presumably, we imperfectly disregard this information, allowing mirrors to be used as decorating features to make small rooms appear larger. Nevertheless, we do understand that the mirrored volume of the room is not the same as the room itself. Similarly, objects seen in a mirror might be accorded less importance when processing the semantic 
meaning of a scene. If mirror information reflects something present in the room, it might be redundant, and if the mirror reflects something from outside the room, it might be irrelevant to the gist of the room.

Here, we analyzed the perception of reflected objects in scenes in two different ways. In Experiment 1, we used a free-viewing labeling task to see whether people label reflected objects in the same manner as they would label nonreflected objects. In Experiment 2, we used a change detection task to check whether the disappearance of an object is as readily detected in a mirror as it is elsewhere in an image.

\section{Experiment 1: Labeling task}

\section{Method}

Participants With no a priori knowledge of the effect size, we aimed for 12 observers, a number that has been adequate to produce meaningful results in prior experiments. Recruiting for 12, we ended up with 11 observers (six females, five males; mean age $=29.5$ years, range $=19-45$ ). All of the observers passed the Ishihara test for color blindness (Ishihara, 1987) and reported normal or corrected-to-normal vision. All procedures were approved by the Partners Healthcare Corporation Institutional Review Board. All observers gave informed consent and were compensated for their time.

Design and procedure Colored images of real-world scenes were obtained from the SUN Database (Xiao, Hays, Ehinger, Oliva, \& Torralba, 2010) and other World Wide Web sources. The stimuli were presented on a 24-in. LCD monitor (resolution $=1,920 \times 1,200$ pixels) that subtended approximately $31^{\circ} \times 23^{\circ}$ of visual angle at a viewing distance of approximately $50 \mathrm{~cm}$. Stimulus presentation and response collection were controlled by MATLAB 7.10.0 and Psychophysics Toolbox 3.0.9 (Brainard, 1997; Pelli, 1997).

Observers were shown 44 pictures of indoor scenes and were asked to label "everything they saw" while they freely viewed one scene at a time and labeled as much or as little as they wanted to, before moving on to the next scene. A subset of 15 images included mirrors that reflected several objects. In ten of the images, 20 objects (e.g., the hairdryer in Fig. 1a) were clearly identifiable both in the room (nonreflected) and as reflections. Objects were excluded if the object in the room was more than 3.3 times bigger in pixel area than the object in the reflection. Several other objects appeared only in the mirror, only in the room, or in both, but not with comparable sizes and/or qualities. These objects are described in later analyses. The remaining 29 scenes served as fillers to prevent observers from guessing our specific interest in mirrors. Observers were encouraged to take no more than 2 min per scene. No feedback was provided. Labeling involved clicking on an object to mark its location and entering a text label to define that object (e.g., "couch").

At the end of the experiment, observers were asked if they had any idea about the purpose of the study, and none of the observers could correctly guess our specific interest in mirrors.

\section{Results}

Figure 1 shows the marks for all observers for two of the images containing mirrors. In Fig. 1a, it can be seen that the hairdryer is labeled by several observers when it is in the room, but not when it is clearly visible in the mirror. Two observers were excluded from this data analysis, since they labeled fewer than $30 \%$ of the images of interest. Observers placed an average of 10.8 labels per image $(S D=1.53)$. For the 20 objects that appeared both in the room and in the mirror with approximately the same size and quality, the objects in the room received an average of 0.8 labels $(S D=0.2)$, whereas the same objects, when reflected in the mirror, received only 0.1 labels on average $(S D=0.2), t(8)=8.87, p<.0001, d=$ $4.0,95 \%$ CI $[0.55,0.93]$ (Fig. 2a).

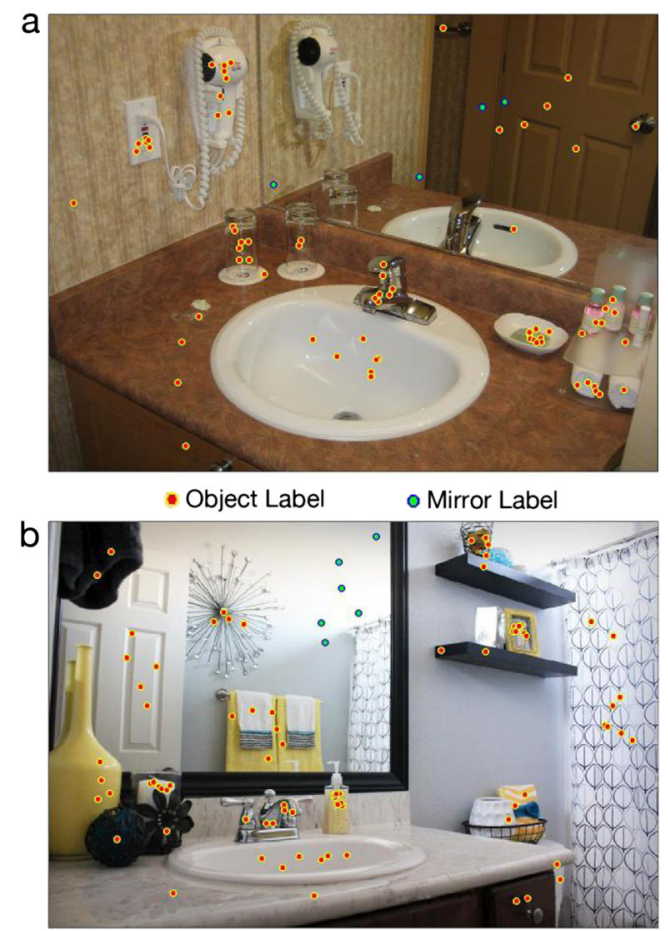

Fig. 1 Pooled labels for all observers on two sample images containing a mirror. (a) The hairdryer, when visible in the room, is labeled by several observers, but not when it is visible in the mirror. (b) When objects appear only in the mirror and are not otherwise visible in the scene (e.g., the towels), they are labeled more often than the reflections of items visible in the room, but still sparsely compared to the nonreflected items. Each individual label is from one observer. Blue dots label the mirror itself 
In all of the scenes containing a mirror, we also identified other objects of interest beyond the 20 well-matched objects. If an object appeared both in the reflection and in the room and either version of the object received at least one label, then the mirror copy was denoted as a "Mirror" object. The corresponding room copy was denoted as the "Room" object. The set of 58 Mirror objects (Fig. 2b Mirror) included the 20 objects analyzed above (plotted in Fig. 2a Mirror), as well as 38 less-well-matched pairs. If an object appeared only in the reflection, it was denoted as "Mirror Only" (total 31 objects), whereas if an object appeared only in the room, it was denoted as "Room Only" (84 objects). Figure 1b shows an example of objects that appeared as reflections but were not otherwise visible in the scene (Mirror Only). As is shown in Fig. 2b, these Mirror Only objects were labeled significantly more often (0.4 labels/object, $S D=0.2$ ) than the reflected objects that were visible in the room as well (Fig. 2b Mirror: 0.0 labels/ object, $S D=0.1), t(8)=4.84, p=.001, d=2.3,95 \% \mathrm{CI}$ $[0.17,0.49]$. However, objects visible only in the mirror were still labeled significantly less often than objects visible only in the room (Mirror Only [0.4 labels/object] vs. Room Only [0.7 labels/object, $S D=0.1]), t(8)=5.44, p=.0006$, $d=2.3,95 \%$ CI $[0.21,0.52]$. Objects that were visible only in the room were labeled as often as the room objects that were also visible in the mirror (Room Only [0.7 labels/ object, $S D=0.1]$ vs. Room [0.7 labels/object, $S D=0.1]$ ), $t(8)=1.85, p=.1, d=0.5,95 \%$ CI $[-0.01,0.11]$. The temporal order of labeling showed that the observers did not label all of the nonreflected objects in an image first before beginning the labeling of reflected objects. The biggest object in the scene was usually labeled first, and this object was frequently reflected in the mirror. The label order of smaller objects was variable.
Discussion

The results of Experiment 1 suggest a devaluing of objects portrayed in mirrors. The failure to mark reflected objects while marking nonreflected versions, when both are visible, might simply be a way to avoid redundancy: Why label the same lamp twice? However, it is instructive that the reflected object was the one that labelers considered redundant. The reflected object in the photograph was treated as less real, even though neither instance was "real" in any physical sense. A desire to avoid redundant labels does not fully explain these results, however, since reflections are still underlabeled relative to nonreflected objects, even when the reflected object is not otherwise visible in the image.

One might propose that the results of Experiment 1 reflect the demand characteristics of the task: Observers were told to label "everything," but were allowed to draw their own conclusions about what that meant. Some observers may have concluded that everything did not include a reflection. In order to get more objective, converging evidence, for Experiment 2 we used a change detection task.

\section{Experiment 2: Change detection}

\section{Method}

Participants Because change detection data are relatively sparse (one data point per image for relatively few images), we doubled our recruitment goal to 24 . Twenty-four observers participated in Experiment 2 (12 females, 12 males; mean age $=28.5$ years, range $=19-51)$ and 30 (16 females, 14 males; mean age $=28.0$ years, range $=20-38)$ in a repetition of the

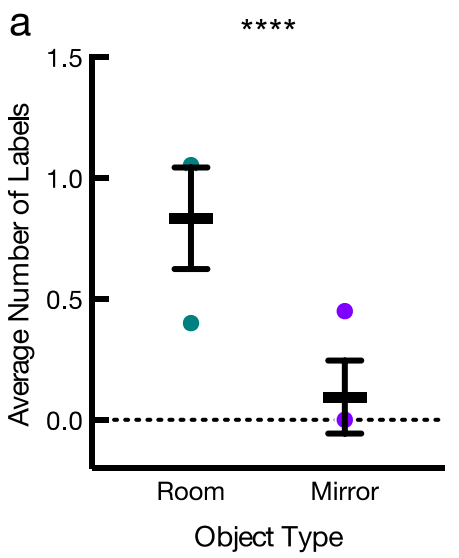

Fig. 2 Comparison of average labels placed on room and mirror objects. (a) Objects in the room (Room) receive more labels than the same objects visible in the mirror (Mirror). (b) When objects are visible only in the mirror (Mirror Only), they are labeled more than the objects visible in both the mirror and room (Mirror). However Mirror Only objects still

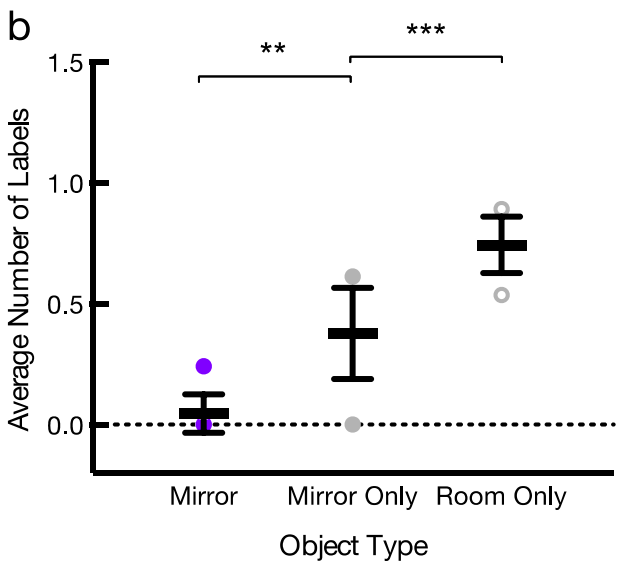

receive fewer labels than objects visible only in the room (Room Only). Means are plotted with standard deviations (SD) as error bars. Minimum and maximum $y$-values are also shown for each case. ${ }^{* *} p \leq .01,{ }^{* * *} p \leq$ $.001,{ }^{* * * * *} p \leq .0001$. Note that the data under (a) Mirror are a subset of the data under (b) Mirror 
experiment. All observers passed the Ishihara test for color blindness (Ishihara, 1987) and reported normal or correctedto-normal vision. All procedures were approved by the Partners Healthcare Corporation Institutional Review Board. All observers gave informed consent and were compensated for their time.

Stimuli and procedure Colored images of real-world scenes were obtained from the SUN Database (Xiao et al., 2010) and other World Wide Web sources. Experiment 2 was carried out at two sites, where the stimulus size varied slightly (Site 1:24in. $\mathrm{LCD}$ monitor, resolution $=1,920 \times 1,200$ pixels, $31^{\circ} \times 23^{\circ}$ at a viewing distance of approximately $50 \mathrm{~cm}$; Site 2: 19-in. monitor, resolution $=1,440 \times 900$ pixels, $32^{\circ} \times 25^{\circ}$ at approximately $50 \mathrm{~cm}$ viewing distance). We found no evidence that this difference had any effect on the results. Moreover, all of the conditions were presented to each observer, and the variations across sites, if any, should be constant in all the conditions, and therefore would not affect the final outcome.

Experiment 2 involved detecting changes in scenes. A series of 109 pairs of images were shown 24 pairs had changes occurring in mirrors, whereas 28 contained mirrors, but the changes in them were elsewhere. As before, the remaining images were fillers with changes at random locations, intended to keep observers from noticing our interest in mirrors.

A flicker paradigm similar to that of Rensink, O'Regan, and Clark (1997) was used. During each trial, an image alternated with a modified version containing a change (presence/absence of an object) with gray blank fields placed between the two images. Each image was displayed for $240 \mathrm{~ms}$ with a $240 \mathrm{~ms}$ blank field in between scenes. The initial view of the image (with or without the changed object) was chosen at random. Observers were asked to press a button as soon as they detected a change and then to click at the location of the change on a static (nonchanging) view of the image; this view was always the version that had the changing object present. A trial ended either when an observer pressed a button to confirm the presence of a change or after $60 \mathrm{~s}$ (timeout), whichever happened first. All trials contained a change. Reaction times were recorded for each keypress and averaged for only the correct responses. All responses with reaction times less than $200 \mathrm{~ms}$ were filtered out.

There were two types of scene conditions, each with a reflected and a nonreflected object change, resulting in four types of change conditions. In the Mirror condition, an object was visible in the room as well as in a reflection (Fig. 3a). The change occurred either to the object in the room or to its reflection. In the disjoint condition, the changing object was either visible in the room or in the mirror, but not simultaneously in both (Fig. 3b). These changes were created using Adobe Photoshop. In order to have controlled backgrounds and contexts for the four types of changes, the same scenes with the same critical objects were used across the scene and change conditions. However, the scenes were distributed between observers in such a way that each observer saw each scene with only one kind of change. Thus, all observers saw all four types of changes, but no scene was repeated for any observer.

At the end of the experiment, observers were asked about the purpose of the study, whether they saw any pattern in the locations of the changes. Over the two experiments, only one observer realized that there were systematic changes in mirrors. This observer did not show a different pattern of results from the average, and the data were retained for analyses.

In the original image set, the sizes of the changing object were similar but not identical in the Mirror condition: That is, reflected objects were generally smaller than their nonreflected versions. To control for this, we repeated this condition with a modified stimulus set. The same images were used, but the sizes of the changed objects were made either smaller or larger in Adobe Photoshop to achieve as small a size difference between the reflected and nonreflected objects as possible, while ensuring sizes that would still look realistic in the scenes.

\section{Results}

The results, shown in Fig. 4, show a penalty for changes presented in a mirror. Due to missing data, one observer was excluded from the Mirror condition and two from the disjoint condition of Experiment 2, because they could not find even a single change when it happened in a reflection. For the same reason, the data from two images were excluded from the analyses of the disjoint condition in Figs. 4 and 5b, and two observers were entirely excluded from the repetition of Experiment 2. Trials that timed out were not analyzed. A higher percentage of trials timed out before observers could detect a change when the change occurred in a reflection (Mirror condition, $14.0 \%$; disjoint condition, $14.0 \%$ ) than when the change occurred in the room (Mirror condition, $6.1 \%$; disjoint condition, $11.4 \%$ ), but this effect was not significant. Note, however, that if these trials were included in the analysis, the difference between the reflected and nonreflected changes would be greater.

Observers were significantly slower to detect changes that occurred in reflections than those that occurred in the nonreflected parts of the scene. This was true in both the Mirror condition, $t(22)=3.89, p=.0008, d=1.1,95 \% \mathrm{CI}$ $[3.10,10.18]$, and the disjoint condition, $t(21)=4.17, p=$ $.0004, d=1.2,95 \%$ CI $[3.24,9.68]$ (Fig 4, left panel).

Though we tried to keep the sizes of the reflected and nonreflected changes roughly equal and the locations of the changes well distributed over the scenes, there were some differences. Figure 5 shows the distributions of the sizes and locations of changes of reflected and nonreflected changing 
a

Mirror Condition: Critical Object

Visible in Both Room \& Mirror

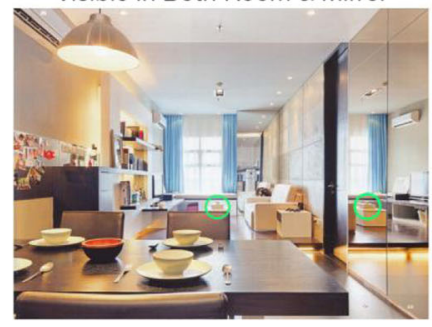

Fig. 3 Sample images showing the types of changes displayed in the change detection task. (a) The Mirror condition comprises changes to objects visible both in the room and in the mirror. (b) The disjoint

b
Disjoint Condition: Critical Object Visible in Either Room or Mirror, not Both

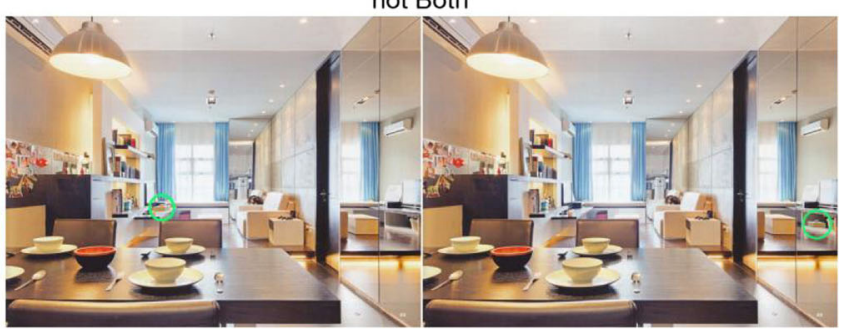

condition has the same object manipulated, but now it is visible either in the room or in the mirror, but not in both. Manipulated object (a book) is encircled in green here for display purposes objects for the mirror (Fig. 5a) and disjoint (Fig. 5b) conditions. Sizes were measured in terms of pixel area. The eccentricities of the targets (measured from the center of the image) did not differ between conditions \{mirror: $t(23)=1.72, p=.1$, $d=0.4,95 \%$ CI $[-117.3,10.65]$; disjoint: $t(21)=1.84, p=$ $.08, d=0.6,95 \%$ CI $[-9.711,162.2]\}$.

Due to the optics of the Mirror condition, the objects in the room were, on average, 1.8 times larger in pixel area than the reflected objects, $t(23)=4.64, p=.0001, d=0.6,95 \% \mathrm{CI}$ $[-1,321,-506.7]$. We observed no significant difference in the sizes of the nonreflected and reflected object changes in the disjoint condition, $t(21)=1.80, p=.09, d=0.3,95 \% \mathrm{CI}$ $[-953.8,68.94]$. Given the significant difference in the sizes of changes in the Mirror condition, we thought that it was reasonable to check whether reflected changes were found later simply because the targets were smaller.

A multiple regression analysis was conducted to evaluate if the change type (i.e., whether the change occurred in the nonreflected or reflected part), the size, and/or the eccentricity of the change predicted reaction time. The results of the

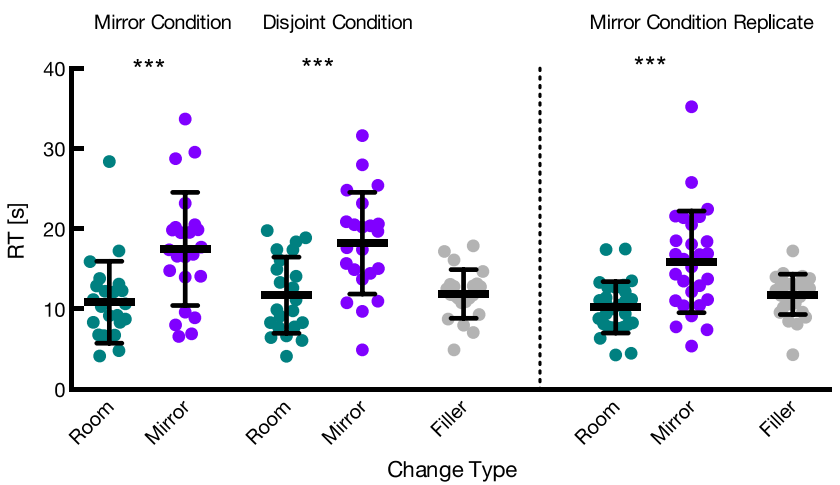

Fig. 4 Average reaction times (RTs) for change detection. In both the mirror and disjoint conditions, changes are detected more slowly when they occur in reflections (Mirror) than when they occur in the nonreflected parts of the scene (Room). The right panel shows the same for the repetition of the Mirror condition, in which there was no difference in the sizes of changed objects in the room and the mirror. Means are plotted with $S D$ as error bars. All individual data points are also shown for each case. ${ }^{* * *} p \leq .001$ multiple regression indicated that for the Mirror condition, the three predictors explained $26.7 \%$ of the variance in reaction time $\left(R^{2}=.27\right.$, adjusted $\left.R^{2}=.22\right), F(3,44)=5.35$, $p=.003$. Size significantly predicted reaction time $(\beta=$ $-.003), t(44)=-3.12, p=.003,95 \%$ CI $[-0.005,-0.001]$, but change type $(\beta=-3.94), t(44)=-1.44, p=.16,95 \% \mathrm{CI}$ $[-9.467,1.591]$, and eccentricity $(\beta=.002), t(44)=0.18, p=$ $.86,95 \%$ CI $[-0.019,0.023]$ did not. For the disjoint condition, the three predictors explained $29.2 \%$ of the variance in reaction time $\left(R^{2}=.29\right.$, adjusted $\left.R^{2}=.24\right), F(3,40)=5.49, p=$ .003. However, for the disjoint condition, only change type
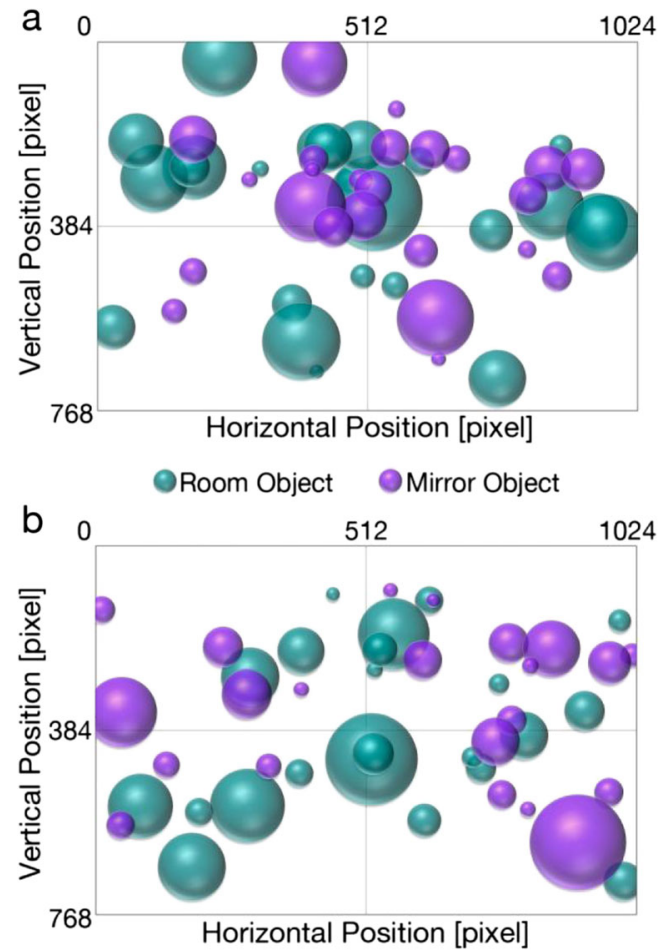

Fig. 5 Illustration of distributions of the sizes and locations of the reflected and nonreflected object changes for the mirror (a) and disjoint (b) conditions. Each bubble is centered at the position of the center of one changing object, and the area of the bubble is proportionate to the area of the object 
predicted the reaction time significantly $(\beta=-8.50), t(40)=$ $-2.99, p=.005,95 \%$ CI $[-14.244,-2.759]$, and not size $(\beta=$ $-.002), t(40)=-1.88, p=.07,95 \%$ CI $[-0.003,0.000]$, or eccentricity $(\beta=.006), t(40)=.53, p=.60,95 \%$ CI $[-0.016$, $0.027]$.

In order to eliminate the size confound in the Mirror condition, we repeated the Mirror condition of Experiment 2 using the same images, altered in Adobe Photoshop, to eliminate significant differences in the nonreflected and reflected change sizes. As in the initial Experiment 2, reaction times were significantly longer, $t(27)=4.31, p=.0002, d=1.1,95 \%$ CI [2.98, 8.37], for the changes in reflected versus nonreflected objects (Fig. 4, right panel). Multiple regression showed that the three predictors explained $32.5 \%$ of the variation in reaction time $\left(R^{2}=.33\right.$, adjusted $\left.R^{2}=.28\right), F(3$, $44)=7.06, p=.0006$. Now, only change type predicted reaction time significantly $(\beta=-6.05), t(44)=-3.21, p=$ $.003,95 \%$ CI $[-9.845,-2.249]$, whereas size $(\beta=-.001)$, $t(44)=-1.97, p=.06,95 \%$ CI $[-0.003,0.000]$, and eccentricity $(\beta=-.012), t(44)=-1.67, p=.11,95 \% \mathrm{CI}[-0.026$, 0.003 ] did not.

Speed and accuracy covaried in this task. Accuracy was always significantly higher for the nonreflected room changes (Table 1, Room Change rows) than for the reflected changes (Table 1, Mirror Change rows) in both the mirror and the disjoint conditions, including in the repetition of the Mirror condition with balanced change size (see Table 1 for the details).

\section{General discussion}

Experiments 1 and 2 provide converging evidence from two very different methods that objects seen in mirrors tend to be perceptually discounted relative to equivalent, nonreflected objects. As was shown in Experiment 1, objects in mirrors glean fewer labels than do objects elsewhere in the image from observers asked to label "everything." Experiment 2 demonstrated that changes to objects in reflections are detected more slowly and less often than changes to nonreflected objects. In some sense, this result might seem obvious. After all, objects in the looking glass are inaccessible in a way that objects in the world are not. That being said, it is worth remembering that all of the objects and all of the changes (reflected or not) in the present experiments were inaccessible, in the sense that they were all simply images presented on a computer screen. It is, perhaps, not so obvious that pixels representing an object in a reflection should be given less weight or attention than pixels representing an object in the nonreflected room. Apparently, parts of images that represent reflections are somewhat less "real" than the rest.

In Experiment 2 and its replicate, we found that people took longer to find changes in a reflection than in a nonreflected part of the scene. It is possible that people do not expect any information to change in a reflection without a corresponding change occurring elsewhere in the scene. After all, the reflection is a copy of the visual information that must be available somewhere else in the scene (although this is not true in photographs taken from a single view). In addition to being redundant for many visual tasks, mirror information is misinformative for certain others, such as determining the shape of a room, planning a route, or reaching for objects. The results from the disjoint condition suggest that people discount mirror information even when the mirror provides information not otherwise present in the scene, indicating that this discounting might be a general strategy that is not easily overcome in a short experiment. It would be interesting to see whether the results would persist after training, or in scenes in which a mirror was the natural place to look for important information-for instance, in the rear-view mirror of a vehicle.

Due to optics, reflected objects in photographs often appear at greater depth than nonreflected objects (for a perceptual contribution to this effect, see Higashiyama \& Shimono,

Table 1 Accuracy of responses

\begin{tabular}{|c|c|c|c|c|c|c|c|}
\hline Condition & $\%$ Accuracy & $S D$ & $t$ & $d f$ & $p$ & $d$ & $95 \% \mathrm{CI}$ \\
\hline \multicolumn{8}{|l|}{ Mirror Condition } \\
\hline Room Change & 83.3 & 18.8 & 2.55 & 22 & .018 & 0.5 & {$[-19.73,-2.01]$} \\
\hline Mirror Change & 72.5 & 21.7 & & & & & \\
\hline \multicolumn{8}{|c|}{ Mirror Condition with Balanced Change Size } \\
\hline Room Change & 82.1 & 20.5 & 3.87 & 27 & .0006 & 0.5 & {$[-16.85,-5.18]$} \\
\hline Mirror Change & 71.1 & 21.6 & & & & & \\
\hline \multicolumn{8}{|l|}{ Disjoint Condition } \\
\hline Room Change & 80.3 & 20.3 & 2.38 & 21 & .027 & 0.6 & {$[-26.95,-1.84]$} \\
\hline Mirror Change & 65.9 & 25.4 & & & & & \\
\hline
\end{tabular}

"Room Change" refers to changes to nonreflected objects, whereas "Mirror Change" refers to changes to reflected objects 
2012). In that context, it is worth noting that we also collected data on objects seen through windows. Of course, such objects also tend to be farther away and less accessible than objects on the viewer's side of the window. We did not find window effects in either our labeling or change detection tasks. Through the looking glass is a different destination than through the window.

Author note P.S. and J.M.W. developed the study concept, and all authors contributed to the study design. P.S. and K.A.E. created the stimuli and collected the data; P.S. analyzed the data; and all authors interpreted and discussed the data. P.S. drafted the manuscript, and K.A.E. and J.M.W. provided critical comments for the revision. All authors approved the final version of the manuscript for submission. This work was supported by German Research Foundation (DFG) Grant No. SA2483/1-1 to P.S. and by Office of Naval Research (ONR) Grant No. N000141010278 and National Institute of Health (NIH) Grant No. F32EY022558 to J.M.W.

\section{References}

Bertamini, M., Latto, R., \& Spooner, A. (2003). The Venus effect: People's understanding of mirror reflections in paintings. Perception, 32, 593-599.

Bertamini, M., Lawson, R., Jones, L., \& Winters, M. (2010). The Venus effect in real life and in photographs. Attention, Perception, \& Psychophysics, 72, 1948-1964. doi:10.3758/APP.72.7.1948

Bertamini, M., Lawson, R., \& Liu, D. (2008). Understanding 2D projections on mirrors and on windows. Spatial Vision, 21, 273-289. doi: $10.1163 / 156856808784532527$
Brainard, D. H. (1997). The Psychophysics Toolbox. Spatial Vision, 10, 433-436. doi:10.1163/156856897X00357

Corballis, M. C. (2001). Why mirrors reverse left and rightCommentary on Navon on mirror reversal. Psycoloquy, 12, 1-4.

Gallup, G. G. J. (1977). Self-recognition in primates: A comparative approach to the bidirectional properties of consciousness. American Psychologist, 32, 327-338.

Higashiyama, A., \& Shimono, K. (2012). Apparent depth of pictures reflected by a mirror: The plastic effect. Attention, Perception, \& Psychophysics, 74, 1522-1532. doi:10.3758/s13414-0120346-4

Ishihara, I. (1987). Ishihara's tests for color-blindness (Conciseth ed.). Tokyo, Japan: Kanehara \& Co.

Navon, D. (2001). The puzzle of mirror reversal: A view from clockland target article on mirror-reversal. Psycoloquy, 12, 1-19.

Navon, D. (2002). It is not all in our mind-Reply to Corballis on Navon on mirror-reversal. Psycoloquy, 13, 1-4.

Pelli, D. G. (1997). The VideoToolbox software for visual psychophysics: Transforming numbers into movies. Spatial Vision, 10, 437-442. doi:10.1163/156856897X00366

Prior, H., Schwarz, A., \& Güntürkün, O. (2008). Mirror-induced behavior in the magpie (Pica pica): Evidence of self-recognition. PLoS Biology, 6, e202. doi:10.1371/journal.pbio.0060202

Proffitt, D. R. (1999). Naive physics. In R. A. Wilson \& F. C. Keil (Eds.), The MIT encyclopedia of the cognitive sciences (pp. 577-579). Cambridge, MA: MIT Press, Bradford Books.

Rensink, R. A., O’Regan, J. K., \& Clark, J. J. (1997). To see or not to see: The need for attention to perceive changes in scenes. Psychological Science, 8, 368-373.

Xiao, J., Hays, J., Ehinger, K. A., Oliva, A., \& Torralba, A. (2010). SUN Database: Large-scale scene recognition from abbey to zoo. In Proceedings of the IEEE Conference on Computer Vision and Pattern Recognition (pp. 3485-3492). Piscataway, NJ: IEEE Press. doi:10.1109/CVPR.2010.5539970 